\title{
Lipophilic efficiency: the most important efficiency metric in medicinal chemistry
} "We propose that employing lipophilic ligand efficiency in conjunction with solid property space analysis can provide clear and
compelling advantages in decision-making for medicinal chemistry."

Keywords: clearance $=$ lipophilic efficiency $\|$ lipophilicity $=$ permeability $\approx$ solubility

Drug discovery in the 'modern' era has gone through many transformations. The science of medicinal chemistry in particular has matured from one heavily dependent on empirical screening of natural products and their derivatives through pharmacological models in the 1950-1970s, to one based on detailed biochemical and mechanistic understanding. This new model was enabled by key technological advances in molecular biology, computers and robotics that arose in the 1980s and early 1990s. This transformed medicinal chemistry into a high-speed enterprise to rapidly produce and test analogs in a timely and low-cost process.

An unanticipated and underappreciated consequence of this new paradigm was an erosion of 'drug-like' physical chemical properties that were critical to success. In the late 1990s, as data sets were compiled to explore broad trends necessary for success, rules began to develop - the most famous of which is the Lipinski 'rule of 5' - that would highlight these physical chemical properties and their relationship to success as an oral drug candidate [1]. Throughout the most recent decade these analyses have been tested, debated, refined and ultimately applied to many more endpoints including predictions related to toxicity and survival in human clinical trials [2-4].

A central theme of these publications is that lipophilicity plays a critical role in medicinal chemistry. Chemists can calculate a host of other physical property values that describe small molecules. These vary from simple attributes such as molecular weight, polar surface area, or number of rotatable bonds to sophisticated descriptors such as atom partial charges, highest occupied molecular orbital energies or molecular volume. While each of these properties can be important in the optimization of compounds in drug-discovery programs, it is also recognized that the application of each is limited to solving specific types of problems. For example, the first-principle relationship between permeability endpoints and the number of hydrogen-bond donors or polar surface area is well appreciated. In contrast, lipophilicity provides a more comprehensive framework to optimize general absorbtion, distribution, metabolism and excretion properties, toxicology profiles and ultimately pharmacological response. Recently there have been numerous reviews that provide excellent connections between lipophilicity and absorbtion, distribution, metabolism and excretion properties that influence success for medicinal chemistry [5-7]. These reviews are far too numerous to cover completely. However, the key parameters that influence success in a standard oral, low-dose drug-discovery program will be addressed. This editorial will briefly examine the strong case for lipophilicity as the dominant consideration for successful medicinal chemistry programs and highlight lipophilic ligand efficiency (LipE), as an important parameter to normalize potency relative to lipophilicity.

A unifying goal in medicinal chemistry is achieving low dose. Lipophilicity directly impacts the dose equation (EQuation I) across several key parameters in multiple orthogonal ways.

$$
\text { Dose } \backsim \frac{(\text { Cefffree } x \text { Clint free })}{\mathrm{F}}
$$

\section{EQuation I}

Absorption is predominantly a function of solubility and permeability. Solubility is perhaps the most basic requirement of an orally available drug, and high lipophilicity dramatically reduces the probability of a given compound achieving acceptable aqueous solubility [8]. Conversely, low lipophilicity can contribute to poor permeability [9]. Taken together, the

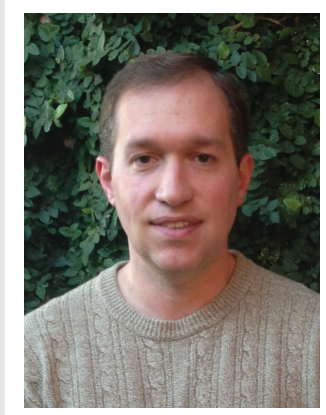

Kevin D Freeman-Cook

Author for correspondence: Worldwide Medicinal Chemistry, 10770 Science Center Drive, San Diego, CA 92121, USA Tel.: +I 8585264844

E-mail: kevin.freeman-cook @pfizer.com

Robert L Hoffman

Worldwide Medicinal Chemistry, 10770 Science Center Drive, San Diego, CA 92I2I, USA

\section{Ted W Johnson}

Worldwide Medicinal Chemistry, 10770 Science Center Drive, San Diego, CA 92I2I, USA 
joint requirements for permeable and soluble compounds imply a fairly narrow range of lipophilicity for most oral drug-discovery programs that are generally within the range of $1.5-3 \operatorname{cog} \mathrm{D}$. Another key aspect of the dose equation is bioavailability that can be improved by avoiding high first pass clearance. Higher lipophilicity positively correlates with clearance, and $\log \mathrm{D}<3$ provides the best opportunity of maintaining favorable metabolic stability [10]. Within closely related chemical structures this relationship is often even more obvious. While not explicitly described in the dose equation, compounds must also possess a suitable safety profile. Recent studies have shown statistically significant improvements in safety outcomes from early in vivo safety studies when compounds have $\operatorname{cog} \mathrm{P}<3$ [4]. These findings are generally consistent with studies that have examined general promiscuity [11]. The sum total of these effects can be seen in the analysis of compounds that survive clinical trials. A recent analysis has shown the mean value of $\operatorname{cog} \mathrm{P}$ for approved drugs has stayed roughly consistent at 2.3-2.6 over a long period of time even as the average $\operatorname{cog} \mathrm{P}$ of drugs in clinical development has increased [12]. This argues strongly for a relationship between attrition and high lipophilicity. This is likely due to a wide variety of factors, some of which have been mentioned in this editorial.

\section{"The careful control of lipophilicity is critical to success in drug discovery."}

For all of the reasons discussed above, the careful control of lipophilicity is critical to success in drug discovery. The key role of lipophilicity is sometimes reflected early in discovery with specific, measurable properties (e.g., clearance, solubility, permeability). At other times, it factors into future studies, which are invariably more expensive (broad off-target screening, animal toxicity or human clinical trials). The other major determinant of success in achieving lowdose compounds (Equation I) is the potency of a given compound against the target of interest. The concept of LipE allows medicinal chemists to normalize the observed potency with changes in lipophilicity [13]. The equation for calculation of LipE is relatively simple (Equation 2). Note that the $\mathrm{K}_{\mathrm{i}}$ or $\mathrm{IC}_{50}$ values should be expressed in molar units. Lipophilicity can be expressed as measured $\log \mathrm{D}$, calculated $\log \mathrm{D}$ or $\operatorname{cog} \mathrm{P}$; while measured values are generally preferred, calculated values are extremely important in prospective design and evaluation of virtual compounds.

$$
\mathrm{Lip} E=-\log \left(\mathrm{K}_{\mathrm{i}} \text { or } \mathrm{IC}_{50}\right)-\log \mathrm{D}(\text { or } \operatorname{cog} \mathrm{P})
$$

Equation 2

The LipE equation mathematically describes the difference between potency and lipophilicity measurements. Lipophilic efficiency can be viewed as a selectivity index between potency and $\log \mathrm{D}$. Specifically, potency is the partitioning to a well-defined target and $\log \mathrm{D}$ represents the $\mathrm{pH}$ dependent partitioning to a nondescript lipophilic environment. Both processes are driven, at least in part for biological targets, by the forces of the hydrophobic effect. LipE increases represent an optimization for the use of not only lipophilicity, but also the polar moieties that define important electrostatic protein-ligand interactions.

“...maximizing lipophilic ligand efficiency in physical property space that best supports good absorption and clearance delivers the highest quality clinical candidates."

Lipophilic efficiency can be used in both early- and late-stage drug-discovery programs. In early triage of chemical matter LipE can be an important parameter to help identify the best lead series. A compelling example for the use of LipE in selecting leads for optimization arises when less potent compounds display higher LipE. The less potent and more lipophilic efficient compounds can provide a better starting point to overlap potency and other desirable properties, while potency-centric views of the data often exclude these less potent compounds as viable leads. Previous measures of efficiency have focused on molecular size and these do not generally account for increased lipophilicity. Potency centric optimization paradigms can increase the difficulty in balancing all of the necessary parameters for achieving safe, low-dose compounds.

LipE continues to play a critical role in the evaluation of specific structural modifications during the progression of a chemical series. Medicinal chemists who employ LipE as a guiding analysis and design concept are able to simultaneously evaluate the effect of a structural change on both potency and lipophilicity. While 
a particular change that improves potency tenfold may appear beneficial, that change may be deceptive if it also increases the lipophilicity of the compound by the same tenfold or one $\log \mathrm{D}$ unit. In this general example the LipE remains unchanged and indicates that no fundamental increase in efficiency was achieved in that particular structural change. Since the additional unit of lipophilicity will increase the forces of the hydrophobic effect between the ligand and any nonaqueous 'target', the observed potency gain for a particular target of interest is less compelling.

LipE increases can be achieved by ligand modifications that impact potency, lipophilicity or both. The largest positive impact on LipE often occurs when modifications improve potency and simultaneously lower lipophilicity. There are both entropic and enthalpic components to increasing LipE. These can involve: optimizing polar and nonpolar interactions; minimizing desolvation penalty; re-enforcingpharmacophoric recognition; releasing highenergy water; minimizing ligand-binding strain and other traditional medicinal chemistry techniques. Although increases in LipE are generally beneficial, consideration of overall physical properties plays a vital role in determining properties of a molecule. Strategies that increase LipE while moving into more desirable property space are ideal, because maximizing LipE in physical property space that best supports

\section{References}

1 Lipinski CA, Lombardo F, Dominy BW, Feeney PJ. Experimental and computational approaches to estimate solubility and permeability in drug discovery and development settings. Adv. Drug Deliv. Rev. 23(1-3), 3-25 (1997).

2 Zhang M-Q, Wilkinson B. Drug discovery beyond the 'rule-of-five'. Curr. Opin. Biotechnol. 18(6), 478-488 (2007).

3 Leeson PD, Springthorpe B. The influence of drug-like concepts on decision-making in medicinal chemistry. Nat. Rev. Drug Discov. 6(11), 881-890 (2007).

4 Hughes JD, Blagg J, Price DA, Bailey S et al. Physicochemical drug properties associated with in vivo toxicological outcomes. Bioorg. Med. Chem. Lett. 18(17), 4872-4875 (2008).

5 Waring MJ. Lipophilicity in drug discovery. Expert Opin. Drug Discov. 5(3), 235-248 (2010). good absorption and clearance delivers the highest quality clinical candidates. Specifically, very polar small molecules $(\log \mathrm{D}<\sim 0)$ may not be ideal drug candidates even if they possess very high LipE, if absorption or active transport-mediated clearance hampers their in vivo performance.

For a medicinal chemist, successfully navigating all of the difficulties in the identification and optimization of lead compounds is an incredible challenge. The overwhelming complexity of drug discovery guarantees that no single parameter can ever fully explain success or failure. Thus, the use of any efficiency metric in isolation is destined to provide only limited predictive insights. However, given the fundamental importance of both potency and lipophilicity, we propose that employing LipE in conjunction with solid property space analysis can provide clear and compelling advantages in decision-making for medicinal chemistry.

\section{Financial \& competing interests disclosure}

The authors have no relevant affiliations or financial involvement with any organization or entity with a financial interest in or financial conflict with the subject matter or materials discussed in the manuscript. This includes employment, consultancies, honoraria, stock ownership or options, expert testimony, grants or patents received or pending, or royalties.

No writing assistance was utilized in the production of this manuscript.
6 van de Waterbeemd H. Physicochemical approaches to drug absorption. In: Drug bioavailability: estimation of solubility, permeability, absorption and bioavailability, Volume 40, 2nd Edition. van de Waterbeemd H, Testa B (Eds). Wiley-VCH Verlag GmbH and Co., Weinheim, Germany, 71-99 (2009).

7 Arnott JA, Planey SL. The influence of lipophilicity in drug discovery and design. Expert Opin. Drug Discov. 7(10), 863-875 (2012).

8 Kramer C, Tilmann H, Fligge T, Beck B, Clark T. A consistent dataset of kinetic solubilities for early-phase drug discovery. ChemMedChem 4(9), 1529-1536 (2009).

9 Waring MJ. Defining optimum lipophilicity and molecular weight ranges for drug candidates-molecular weight dependent lower $\log \mathrm{D}$ limits based on permeability. Bioorg. Med. Chem. Lett. 19(10), 2844-2851 (2009).
10 Johnson TW, Dress KR, Edwards M. Using the golden triangle to optimize clearance and oral absorption. Bioorg. Med. Chem. Lett. 19(19), 5560-5564 (2009).

11 Peters J-U, Schnider P, Mattei P, Kansy M. Pharmacological promiscuity: dependence on compound properties and target specificity in a set of recent Roche compounds. ChemMedChem 4(4), 680-686 (2009).

12 Leeson PD, Davis AM. Time-related differences in the physical property profiles of oral drugs. J. Med. Chem. 47(25), 6338-6348 (2004).

13 Edwards MP, Price DA. Role of physicochemical properties and ligand lipophilicity efficiency in addressing drug safety risks. Ann. Reports Med. Chem. 45, 381-391 (2010). 